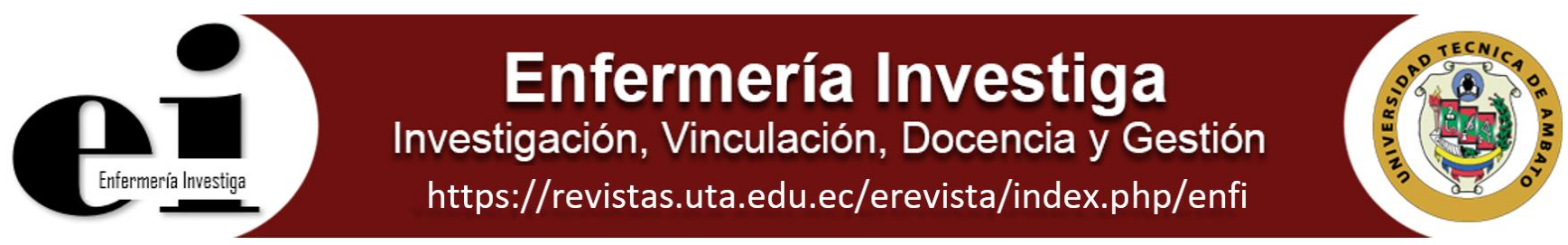

\title{
ANÁLISIS DE LA ATENCIÓN DEL USUARIO EN LOS CENTROS DE SALUD DE LA PROVINCIA DE MANABÍ-ECUADOR.
} ANALYSIS OF USER CARE IN HEALTH CENTERS IN THE PROVINCE OF MANABÍ-ECUADOR.

\author{
Carlos García Escovar ${ }^{1}$ 0000-0003-2436-9497, Leobaldo Barrera Paz² 0000-0003-4480-0416
}

${ }^{1}$ Docente y Miembro de la Comisión de Investigación de la Facultad de Ciencias Médicas de la ULEAM, Ecuador, Doctorante de Ciencias Médicas de la División de Estudios para Graduados, Facultad de Medicina, Universidad del Zulia, Venezuela

${ }^{2}$ Doctorado de Ciencias Médicas, División de Estudios para Graduados, Facultad de Medicina, Universidad del Zulia, Venezuela

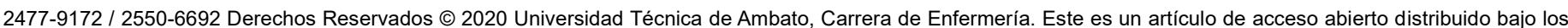

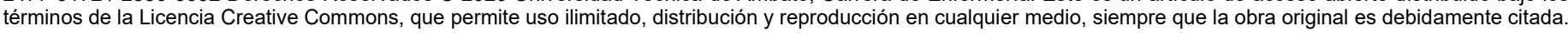

Recibido: 01 de septiembre 2020

Aceptado: 25 de noviembre2020

\section{RESUMEN}

Introducción: La calidad del servicio de salud está relacionado con la satisfacción del usuario, es importante conocer su percepción, para lograr una atención de calidad. Objetivo: analizar la atención del usuario en los centros de salud de la Provincia de Manabí-Ecuador. Método: Estudio de campo, descriptivo y transversal, con una muestra no probabilista de 924, mayores de 20 años, de ambos sexos, durante el año 2019, se aplicó la Encuesta Nacional de Satisfacción de Usuarios en Salud, del Instituto Nacional de Estadística e Informática del Ministerio de Salud de Perú, modificada y adaptada según los indicadores de las siguientes variables: sexo, edad, procedencia, realidad socioeconómica, asistencia a instituciones de salud, enfermedades crónicas, servicios de salud utilizados, tiempo de espera para asignación de consultas y calidad de atención. Resultados: Se encontró predominio del sexo femenino y la edad entre 20 a 39 años, el Ministerio de Salud Pública y el Instituto Ecuatoriano de Seguridad Social fueron las instituciones más utilizadas, la diabetes e hipertensión arterial son las enfermedades crónicas más frecuentes y los servicios más requerido fueron medicina general, las especialidades, el laboratorio y radiología, predominó la atención inmediata en medicina general y el tiempo más prolongado de 1 semana en el servicio de promoción/prevención de enfermedades y la calidad de la atención fue buena en más del $50 \%$. Conclusiones: la mayoría de los usuarios son adultos jóvenes, con enfermedades crónicas de alta morbi-mortalidad en el país, quienes utilizan los servicios públicos y la atención recibida fue percibida como satisfactoria.

Palabras Clave: usuario de salud, servicios de salud, satisfacción en salud.

\section{ABSTRACT}

Introduction: The quality of the health service is related to user satisfaction, it is important to know their perception in order to achieve quality care. Objective: to analyze user care in health centers in the Province of Manabí-Ecuador. Method: Descriptive and cross-sectional field study, with a non-probabilistic sample of 924, over 20 years of age, of both sexes, during 2019, the National Survey of User Satisfaction in Health, from the National Institute of Statistics and Information technology of the Ministry of Health of Perú, modified and adapted according to the indicators of the following variables: sex, age, origin, socioeconomic reality, attendance at health institutions, chronic diseases, health services used, waiting time for appointment of consultations and attention quality. Results: A predominance of the female sex and age between 20 to 39 years was found, the Ministry of Public Health and the Ecuadorian Institute of Social Security were the most used institutions, diabetes and arterial hypertension are the most frequent chronic diseases and the most frequent services required were general medicine, specialties, laboratory and radiology, immediate care prevailed in general medicine and the longest time of 1 week in the disease promotion / prevention service and the quality of care was good in more than $50 \%$. Conclusions: the majority of users are young adults, with chronic diseases of high morbidity and mortality in the country, who use public services and the care received was perceived as satisfactory.

\section{Keywords: user, health services, Ecuador}




\section{INTRODUCCIÓN}

Toda institución de salud pretende lograr la excelencia en el servicio y se interesa por conocer la calidad, satisfacción o percepción que tienen los usuarios (pacientes), sobre la atención prestada. En los primeros centros hospitalarios se inician las investigaciones que permitieron tener un mayor conocimiento sobre las enfermedades que se presentaban y diezmaban a la población, como ocurrió con las epidemias, conllevando al mejoramiento de los métodos científicos y de los servicios $(1,2)$, que produjeron cambios importantes en los sistemas de salud en múltiples regiones del mundo, adecuando los servicios sanitarios a las necesidades de los pacientes y Ecuador no escapa a estos cambios (3)

El órgano rector de la salud es el Sistema Nacional de Salud (SNS) y tiene entre sus funciones el financiamiento, aseguramiento y provisión de sus servicios, garantizando un servicio de salud de calidad a todas las personas, en cualquier lugar y en el momento que se requiera, independientemente de la estructura institucional que se posea en cada país (4). Este servicio puede estar integrado por varios proveedores configurando subsistemas dentro del SNS y dependiendo de las características sociales, económicas y políticas de cada país, será diferente (5), así en Latinoamérica la característica que se observa con más frecuencia es una estructura altamente segmentada y fragmentada (6).

En ese sentido, el Sistema de Salud en Ecuador (SSE) ha sufrido importantes transformaciones debido a los cambios políticos, económicos y sociales ocurridos en las dos últimas décadas, con la Constitución de la República del 2008, se establece el nuevo Sistema Nacional de Salud Pública, gratuito en todos los niveles, integrado en el seguro universal obligatorio; cuyo objetivo es la atención integral del individuo, bajo la rectoría del Ministerio de Salud Pública (MSP) como Autoridad Sanitaria Nacional (7). No obstante, este sistema está compuesto por dos sectores, el público y el privado, es segmentado debido a la coexistencia de subsistemas con financiamiento, y afiliación, que atienden a diferentes grupos poblacionales, con barreras de acceso; y fragmentado por la coexistencia de diversas unidades no integradas a la red de los servicios de salud (5), por ello, se ha propuesto la integración de los sistemas para lograr mejorar su desempeño (6).

EI SSE perteneciente al sector público está conformado por el Ministerio de Salud Pública, que da servicios a todos los ciudadanos, el Ministerio de Inclusión Económica y Social (MIES) y los Servicios de Salud de las municipalidades, ambos con programas y establecimientos de salud, que también atienden a las personas no aseguradas, las Instituciones de Seguridad Social: el Instituto Ecuatoriano de Seguridad Social (IESS), los Institutos de Seguridad Social de las Fuerzas Armadas (ISSFA) y de la Policía Nacional (ISSPOL), mientras que el sector privado está integrado por organizaciones con fines de lucro como hospitales, clínicas, dispensarios, consultorios, farmacias e instituciones de medicina prepagada, así como otras de la sociedad civil y de servicio social sin fines lucrativos (8).

EI MSP de Ecuador tiene como objetivo la atención integral del usuario en las diferentes instituciones sanitarias bajo su rectoría, que se define como actividades interrelacionadas que se ofrecen a un usuario o cliente por un suministrador, con la finalidad de proporcionar un producto en el momento y lugar adecuado, haciendo de este un uso correcto (8), en el caso de salud el usuario es el paciente. En ese sentido, se conocen los resultados de investigaciones sobre la calidad y satisfacción de los usuarios de los servicios de salud en Ecuador y en otros países de Latinoamérica, reportando valores diferentes entre ellos (2), (912).

Al respecto, la calidad de un servicio se relaciona con la satisfacción del usuario según su interés o necesidad (13), se hace necesario su evaluación a través de la información, que genere el usuario sobre sus expectativas y percepción del servicio que recibe, es decir, entre lo que espera y lo que percibe sobre el producto recibido, lo que permitirá realizar el mejoramiento de los mismos (14); para el sector salud no existe una definición consensuada, pero igualmente se vincula a la satisfacción del paciente (15) y para su evaluación se emplean instrumentos que miden la interacción del usuario con el sistema, desde el punto de vista técnico (asistencia, avances científicos y capacitación de los profesionales) y humano-ambiental o subjetiva, esta última evalúa la satisfacción sin incluir los aspectos clínicos (2). Todo lo anteriormente descrito lleva a considerar la necesidad de realizar estudios sobre la percepción que tienen los usuarios sobre los servicios de salud, que reciben en los centros asistenciales a los cuales acuden, dada la obligación que tienen estas instituciones de proporcionar calidad en la atención de los pacientes (2), por estas razones el presente trabajo tiene el objetivo analizar la atención del usuario en los centros de salud de la Provincia de Manabí-Ecuador.

\section{MÉTODO}

La muestra fue no probabilista y para disminuir el sesgo se incluyeron al azar los usuarios que asistían a las instituciones de salud y en espacios públicos abiertos de la provincia de Manabí de Manta, Portoviejo y Montecristi. Un total de 924 personas aceptaron contribuir en este estudio, de 20 años o más, de ambos sexos, distribuidos de la siguiente manera: Manta: 585 (63,31\%), Portoviejo: 180 (19,48\%) y Montecristi: 159 (17,21\%), y se les informó sobre el objetivo y alcance que se perseguía con el mismo y se les solicitó su consentimiento por escrito.

El instrumento utilizado para analizar la atención del usuario fue la Encuesta Nacional de Satisfacción de Usuarios en Salud, del Instituto Nacional de Estadística e Informática del Ministerio de Salud de Perú (17), la cual fue modificada y adaptada según los indicadores de las variables de este estudio, seguidamente se sometió a validación a través del juicio de expertos. En esta se recoge información general sobre la edad, sexo, lugar de residencia e ingreso familiar para clasificarlos según el grupo socioeconómico a la cual pertenecían (16), y de manera específica la afiliación a un servicio de salud, padecimiento de enfermedad crónica, servicio utilizado en los últimos 6 meses, el tiempo de espera para la asignación de la cita y satisfacción con la atención recibida en el servicio (17). El rango de edad en años que se consideró fue el establecido por el MSP de Ecuador de la siguiente manera: 20-39 (adulto joven), 40- 64 (adulto) y más de 65 (adulto mayor) (18).

A cada uno de los participantes se les realizó una entrevista con las preguntas contenidas en la encuesta antes descrita, de carácter anónima y se especificó sobre la confidencialidad de esta; el participante, previa lectura, decidía si responderla o no.

Una vez culminado el proceso de recolección de la información en las encuestas aplicadas, se procedió a analizar los resultados de acuerdo los indicadores de las variables estudiadas, por 
medio de un análisis estadístico inferencial aplicando a través del Chi Cuadrado con SPSS Estatistics 20.0 para windows. Los resultados se presentan en tablas, expresados en promedio y desviación estándar, considerando significancia estadística una $p<0.05$.
Los datos obtenidos muestran que de los 924 usuarios participantes en este estudio predominó el sexo femenino $(56,28 \%)$ y el promedio de edad fue $33 \pm 13,25$ años, el grupo de adultos más atendido correspondió a los adultos jóvenes $(72,07 \%)$ y la condición socioeconómica más frecuente fue "no pobre" $(97,19 \%)$ (tabla 1).

\section{RESULTADOS}

TABLA 1

\begin{tabular}{lc}
$\begin{array}{c}\text { CARACTERISTICAS SOCIODEMOGRÁFICAS DE LOS USUARIOS } \\
\text { QUE ASISTEN A LOS CENTROS DE SALUD DE ECUADOR }\end{array}$ \\
\hline Parámetros & Número de Casos (Porcentaje) \\
\hline Sexo: & $404(43,72 \%)$ \\
Masculino & $520(56,28 \%)$ \\
Femenino & \\
Edad (años): & $666(72,07 \%)$ \\
$20-39$ & $228(24,68 \%)$ \\
$40-64$ & $30(3,25)$ \\
$\geq 65$ & \\
Condición & $10(1,08 \%)$ \\
socioeconómica: & $16(1,73 \%)$ \\
Indigente & $898(97,19 \%)$ \\
Pobre & \\
No pobre &
\end{tabular}

En relación la afiliación a las instituciones prestadoras de servicio de salud de Ecuador, se encontró que la mayor frecuencia fue para el sector público encabezado por el MSP
$(43,67 \%)$ seguido por el IESS $(38,46 \%)$, la medicina Pre-pagada $(12,77 \%)$ y la atención privada $(11,79 \%)$ (tabla 2$)$.

TABLA 2

\begin{tabular}{|c|c|c|}
\hline $\begin{array}{c}\text { INSTITUCIONES PRESTADORES DE SERVICIO } \\
\text { DE SALUD }\end{array}$ & $\begin{array}{l}\text { NÚMERO DE } \\
\text { CASOS }\end{array}$ & PORCENTAJE \\
\hline Ministerio de Salud Pública & 352 & 43,67 \\
\hline Instituto Ecuatoriano de Seguridad Social & 310 & 38,46 \\
\hline Medicina Pre-pagada & 98 & 12,77 \\
\hline Atención Privada & 95 & 11,79 \\
\hline $\begin{array}{l}\text { Instituto de Seguridad Social de las Fuerzas } \\
\text { Armadas }\end{array}$ & 20 & 2,48 \\
\hline Gobierno Autónomo Descentralizado & 12 & 1,49 \\
\hline Medicina Familiar & 5 & 0,62 \\
\hline Entidad Religiosa & 4 & 0,49 \\
\hline ONG Fundación & 3 & 0,37 \\
\hline $\begin{array}{l}\text { Instituto de Seguridad Social de la Policia } \\
\text { Nacional }\end{array}$ & 3 & 0,37 \\
\hline Junta Beneficencia & 2 & 0,25 \\
\hline
\end{tabular}

La distribución de las enfermedades crónicas no transmisibles según el sexo y la edad se evidencian mayor frecuencia en la diabetes mellitus (DM), hipertensión arterial (HTA) y la obesidad, tanto en el sexo femenino y masculino, sin diferencias 
significativas; no obstante, la enfermedad tiroidea y el renglón de otras enfermedades fue significativamente mayor en las mujeres $(p<0,05)$. Al analizar estos parámetros según el rango de edad se encontró que los adultos de 40-64 años evidenciaron una diferencia significativa $p<0,05$ en la DM, HTA, obesidad, infarto al miocardio, artrosis y discapacidad mental (tabla 3 ). TABLA 3

ENFERMEDAD CRÓNICA NO TRANSMISIBLE, SEGÚN EL SEXO Y LA EDAD

\begin{tabular}{|c|c|c|c|c|}
\hline \multirow{2}{*}{$\begin{array}{l}\text { Enfermedad } \\
\text { Crónica }\end{array}$} & \multicolumn{2}{|c|}{$\begin{array}{c}\text { Sexo } \\
n=924\end{array}$} & \multicolumn{2}{|c|}{$\begin{array}{l}\text { Edad Adulta (años) } \\
\qquad n=924\end{array}$} \\
\hline & $\begin{array}{c}\text { Femenino } \\
n=520 \\
(56,28 \%)\end{array}$ & $\begin{array}{c}\text { Masculino } \\
n=404 \\
(43,72 \%)\end{array}$ & $\begin{array}{c}\text { Joven } \\
(20-39) \\
n=666(72,07 \%)\end{array}$ & $\begin{array}{c}\text { Adulto } \\
(40-64) \\
\mathrm{n}=228(24,68 \%)\end{array}$ \\
\hline Sindrome Metabólico & $24(4,62 \%)$ & $16(3,96 \%)$ & $24(3,60 \%)$ & $13(5,70 \%)$ \\
\hline Diabetes Mellitus & $112(21,54 \%)$ & $73(18,07 \%)$ & $123(18,47 \%)$ & $47(20,61 \%)^{\star}$ \\
\hline Hipertensión Arterial & $135(25,96 \%)$ & $85(21,04 \%)$ & $133(19,97 \%)$ & $73(32,02 \%)^{\star}$ \\
\hline Dislipidemia & $25(4,81 \%)$ & $14(3,47 \%)$ & $25(3,75 \%)$ & $10(4,39 \%)$ \\
\hline Bajo Peso & $18(3,46 \%)$ & $14(3,47 \%)$ & $25(3,75 \%)$ & $5(2,19 \%)$ \\
\hline Obesidad & $76(14,62 \%)$ & $47(11.63 \%)$ & $74(11,11 \%)$ & $43(18,86 \%)^{\star}$ \\
\hline Enfermedad Tiroidea & $40(7,69 \%)^{*}$ & $17(4,21 \%)$ & $33(4,95 \%)$ & $21(9,21 \%)$ \\
\hline Infarto al Miocardio & $14(2,69 \%)$ & $10(2,48 \%)$ & $13(1,95 \% \%)$ & $8(3,51 \%)^{*}$ \\
\hline Enfermedad Cerebrovascular & $6(1,15 \%)$ & $5(1,24 \%)$ & $8(1,20 \%)$ & $3(1,32 \%)$ \\
\hline Artrosis & $51(9,81 \%)$ & $34(8,42 \%)$ & $48(7,21 \%)$ & $31(13,59 \%)^{*}$ \\
\hline Discapacidad Fisica & $34(6,54 \%)$ & $24(5,94 \%)$ & $37(5,55 \%)$ & $18(7,89 \%)$ \\
\hline Discapacidad Mental & $22(4,23 \%)$ & $9(2,23 \%)$ & $18(2,70 \%)$ & $12(5,26 \%)^{*}$ \\
\hline Tabaquismo & $27(5,19 \%)$ & $22(5,45 \%)$ & $39(5,86 \%)$ & $9(3,95 \%)$ \\
\hline Alcoholismo & $29(5,58 \%)$ & $22(5,45 \%)$ & $40(6,01 \%)$ & $8(3,51 \%)$ \\
\hline Otras adicciones & $8(1,54 \%)$ & $4(0,99 \%)$ & $11(1,65 \%)$ & $1(0,44 \%)$ \\
\hline Otras & $57(10,96 \%)^{*}$ & $28(6,93 \%)$ & $58(8,71 \%)$ & $24(10,53 \%)$ \\
\hline
\end{tabular}

En los servicios de atención médica de las instituciones de salud de Ecuador utilizados por los usuarios en los últimos seis meses, según el sexo y la edad, se evidencia que la asistencia al Médico General, Médico Especialista y al Laboratorio o Radiología fueron los servicios más empleados por los pacientes, con valores similares para ambos sexos y sin diferencias significativas entre ellos, igualmente cuando se discrimina por edad, para la medicina general los valores fueron ligeramente superior al $70 \%$ en todos los rangos de edad, mientras que la atención por el médico especialista fue superior al $50 \%$ en los mayores de 40 años y significativamente menor para los adultos jóvenes cuya edad se encuentra entre los 20 a 39 años, pero los servicios de laboratorio/radiología fue superior en los adultos mayores sin diferencias estadísticamente significativas entre ellos (tabla 4)

TABLA 4

SERVICIOS DE ATENCIÓN MÉDICA UTILIZADOS POR LOS USUARIOS EN LAS INSTITUCIONES DE SALUD DE ECUADOR, SEGÚN SEXO Y EDAD.

\begin{tabular}{|c|c|c|c|c|c|}
\hline \multirow[b]{2}{*}{$\begin{array}{l}\text { Servicios } \\
\text { de } \\
\text { Salud }\end{array}$} & \multicolumn{2}{|c|}{$\begin{array}{c}\text { Sexo } \\
n=924\end{array}$} & \multicolumn{3}{|c|}{$\begin{array}{l}\text { Edad (años) } \\
\quad n=924\end{array}$} \\
\hline & $\begin{array}{c}\text { Femenino } \\
n=520 \\
(56,28 \%)\end{array}$ & $\begin{array}{c}\text { Masculino } \\
n=404 \\
(43,72 \%)\end{array}$ & $\begin{array}{c}\text { Adulto Joven } \\
20-39 \\
n=666\end{array}$ & $\begin{array}{c}\text { Adulto } \\
40-64 \\
n=228\end{array}$ & $\begin{array}{c}\text { Adulto Mayor } \\
>65 \\
n=30\end{array}$ \\
\hline $\begin{array}{c}\text { Medicina General } \\
n=661(71,54 \%)\end{array}$ & $\begin{array}{c}374 \\
(71,92 \%)\end{array}$ & $\begin{array}{c}287 \\
(71,04 \%)\end{array}$ & $\begin{array}{c}474 \\
(71,17 \%)\end{array}$ & $\begin{array}{c}165 \\
(72,36 \%)\end{array}$ & $\begin{array}{c}22 \\
(73,33 \%)\end{array}$ \\
\hline $\begin{array}{l}\text { Médico Especialista } \\
n=392(41,42 \%)\end{array}$ & $\begin{array}{c}228 \\
(43,85 \%)\end{array}$ & $\begin{array}{c}164 \\
(40,59 \%\end{array}$ & $\begin{array}{c}261^{*} \\
(39,19 \%)\end{array}$ & $\begin{array}{c}115 \\
(50,44 \%)\end{array}$ & $\begin{array}{c}16 \\
(53,33 \%)\end{array}$ \\
\hline $\begin{array}{l}\text { Laboratorio/Radiología } \\
\qquad \mathrm{n}=394(42,64 \%)\end{array}$ & $\begin{array}{c}220 \\
(42,31 \%)\end{array}$ & $\begin{array}{c}174 \\
(43,07 \%)\end{array}$ & $\begin{array}{c}281 \\
(42,19 \%)\end{array}$ & $\begin{array}{c}98 \\
(42,98 \%)\end{array}$ & $\begin{array}{c}15 \\
(50 \%)\end{array}$ \\
\hline $\begin{array}{c}\text { Odontología } \\
n=223(24,13 \%)\end{array}$ & $\begin{array}{c}122 \\
(23,46 \%)\end{array}$ & $\begin{array}{c}101 \\
(25 \%)\end{array}$ & $\begin{array}{c}153 \\
(22,97 \%)\end{array}$ & $\begin{array}{c}60 \\
(26,32 \%)\end{array}$ & $\begin{array}{c}10 \\
(33,33 \%)\end{array}$ \\
\hline $\begin{array}{c}\text { Urgencia } \\
n=346(37,45 \%)\end{array}$ & $\begin{array}{c}193 \\
(37,12 \%)\end{array}$ & $\begin{array}{c}153 \\
(37,87 \%)\end{array}$ & $\begin{array}{c}254 \\
(38,1 \%)\end{array}$ & $\begin{array}{c}83 \\
(36,40 \%)\end{array}$ & $\begin{array}{c}9 \\
(30 \%)\end{array}$ \\
\hline $\begin{array}{l}\text { Hospitalización/Cirugía } \\
n=128(13,85 \%)\end{array}$ & $\begin{array}{c}79 \\
(15,19 \%)\end{array}$ & $\begin{array}{c}49 \\
(12,13 \%)\end{array}$ & $\begin{array}{c}86 \\
(12,91 \%)\end{array}$ & $\begin{array}{c}35 \\
(15,35 \%)\end{array}$ & $\begin{array}{c}7 \\
(23,33 \%)\end{array}$ \\
\hline $\begin{array}{c}\text { Promoción/Prevención de } \\
\text { Enfermedades } \\
n=199(21,54 \%)\end{array}$ & $\begin{array}{c}112 \\
(21,54 \%)\end{array}$ & $\begin{array}{c}87 \\
(21,53 \%)\end{array}$ & $\begin{array}{c}139 \\
(20,87 \%)\end{array}$ & $\begin{array}{c}51 \\
(22,37 \%)\end{array}$ & $\begin{array}{c}9 \\
(30 \%)\end{array}$ \\
\hline $\begin{array}{c}\text { Fisioterapia } \\
n=97(10,17 \%)\end{array}$ & $\begin{array}{c}51 \\
(9,81 \%)\end{array}$ & $\begin{array}{c}46 \\
(11,34 \%)\end{array}$ & $\begin{array}{c}67 \\
(10,06 \%)\end{array}$ & $\begin{array}{c}26 \\
(11,40 \%)\end{array}$ & $\begin{array}{c}4 \\
(13,33 \%)\end{array}$ \\
\hline
\end{tabular}

En relación al tiempo que debieron esperar los usuarios para ser atendidos por los servicios requeridos, predominó la atención inmediata en medicina general y el tiempo más prolongado de 1 semana en el servicio de promoción/prevención de enfermedades (tabla 5). 
TABLA 5

\begin{tabular}{|c|c|c|c|c|c|}
\hline \multirow{2}{*}{$\begin{array}{c}\text { Servicios } \\
\text { Médicos } \\
\mathrm{n}=924(100 \%)\end{array}$} & \multicolumn{5}{|c|}{ Tiempo (Semanas) } \\
\hline & Inmediato & 1 & 2 & 3 & $>4$ \\
\hline $\begin{array}{c}\text { Medicina General } \\
n=661 \\
(71,54 \%)\end{array}$ & $\begin{array}{c}216 \\
(32,68 \%)\end{array}$ & $\begin{array}{c}235 \\
(35,55 \%)\end{array}$ & $\begin{array}{c}140 \\
(21,18 \%)\end{array}$ & $\begin{array}{c}20 \\
(3,03 \%)\end{array}$ & $\begin{array}{c}50 \\
(7,56 \%)\end{array}$ \\
\hline $\begin{array}{c}\text { Médico Especialista } \\
n=392 \\
(41,42 \%)\end{array}$ & $\begin{array}{c}119 \\
(30,36 \%)\end{array}$ & $\begin{array}{c}143 \\
(36,48 \%)\end{array}$ & $\begin{array}{c}60 \\
(15,31 \%)\end{array}$ & $\begin{array}{c}17 \\
(4,34 \%)\end{array}$ & $\begin{array}{c}53 \\
(13,92 \%)\end{array}$ \\
\hline $\begin{array}{l}\text { Laboratorio/ } \\
\text { Radiologia } \\
n=394 \\
(42,64 \%)\end{array}$ & $\begin{array}{c}134 \\
(34,01 \%)\end{array}$ & $\begin{array}{c}150 \\
(38,07 \%)\end{array}$ & $\begin{array}{c}54 \\
(13.71 \%)\end{array}$ & $\begin{array}{c}19 \\
(4,82 \%)\end{array}$ & $\begin{array}{c}37 \\
(9,39 \%)\end{array}$ \\
\hline $\begin{array}{l}\text { Odontología } \\
n=223 \\
(24,13 \%)\end{array}$ & $\begin{array}{c}85 \\
(38,11 \%)\end{array}$ & $\begin{array}{c}78 \\
(34,98 \%)\end{array}$ & $\begin{array}{c}38 \\
(17,04 \%)\end{array}$ & $\begin{array}{c}13 \\
(5,83 \%)\end{array}$ & $\begin{array}{c}9 \\
(4,04 \%)\end{array}$ \\
\hline $\begin{array}{c}\text { Promoción/Prevención de } \\
\text { Enfermedades } \\
n=199 \\
(21,54 \%)\end{array}$ & $\begin{array}{c}58 \\
(29,15 \%)\end{array}$ & $\begin{array}{c}112 \\
(56,28 \%)\end{array}$ & $\begin{array}{c}27 \\
(13,57 \%)\end{array}$ & $\begin{array}{c}2 \\
(1,01 \%)\end{array}$ & 0 \\
\hline $\begin{array}{c}\text { Hospitalización/Cirugia } \\
n=128 \\
(13,85 \%)\end{array}$ & $\begin{array}{c}48 \\
(37,50 \%)\end{array}$ & $\begin{array}{c}36 \\
(28,13 \%)\end{array}$ & $\begin{array}{c}17 \\
(13,28 \%)\end{array}$ & $\begin{array}{c}17 \\
(13,28 \%)\end{array}$ & $\begin{array}{c}10 \\
(7,81 \%)\end{array}$ \\
\hline $\begin{array}{c}\text { Fisioterapia } \\
n=97 \\
(10,17 \%)\end{array}$ & $\begin{array}{c}31 \\
(31,96 \%)\end{array}$ & $\begin{array}{c}40 \\
(44,44 \%)\end{array}$ & $\begin{array}{c}15 \\
(15,46 \%)\end{array}$ & $\begin{array}{c}7 \\
(7,22 \%)\end{array}$ & $\begin{array}{c}4 \\
(4,12 \%)\end{array}$ \\
\hline
\end{tabular}

$\mathrm{n}=$ representa número de casos

En la percepción que tienen los usuarios sobre la calidad de los servicios recibida en las instituciones de salud donde fueron atendidos, correspondiendo a la categoría buena en medicina general $(41,91 \%)$, medicina especialista $(44,90)$, laboratorio/radiología (42,89\%), odontología $\quad(66,37 \%)$, Programas de Promoción y Prevención (51,26\%), hospitalización/cirugía (50\%) y fisioterapia (24,74\%) (tabla 6).

Tabla 6

PERCEPCIÓN DE LOS USUARIOS SOBRE LA CALIDAD DE LA ATENCIÓN MEDICA RECIBIDA EN LAS INSTITUCIONES DE SALUD DE ECUADOR

\begin{tabular}{|c|c|c|c|c|c|}
\hline \multirow{2}{*}{$\begin{array}{l}\text { Servicios } \\
\text { Médicos }\end{array}$} & \multicolumn{5}{|c|}{ Percepción de la Calidad del Servicio } \\
\hline & Muy Mala & Mala & Ni Mala Ni Buena & Buena & Excelente \\
\hline $\begin{array}{c}\text { Medicina General } \\
n=661 \\
(71,54 \%)\end{array}$ & $\begin{array}{c}29 \\
(4,39 \%)\end{array}$ & $\begin{array}{c}44 \\
(6,66 \%)\end{array}$ & $\begin{array}{c}239 \\
(36,16 \%)\end{array}$ & $\begin{array}{c}277 \\
(41,91 \%)\end{array}$ & $\begin{array}{c}72 \\
(10,88 \%)\end{array}$ \\
\hline $\begin{array}{c}\text { Médico Especialista } \\
n=392 \\
(41,42 \%)\end{array}$ & $\begin{array}{c}13 \\
(3,32 \%)\end{array}$ & $\begin{array}{c}13 \\
(3,32 \%)\end{array}$ & $\begin{array}{c}107 \\
(27,30 \%)\end{array}$ & $\begin{array}{c}176 \\
(44,90 \%)\end{array}$ & $\begin{array}{c}83 \\
(21,16 \%)\end{array}$ \\
\hline $\begin{array}{c}\text { Laboratorio/ } \\
\text { Radiología } \\
n=394 \\
(42,64 \%)\end{array}$ & $\begin{array}{c}19 \\
(4,82 \%)\end{array}$ & $\begin{array}{c}22 \\
(5,58 \%)\end{array}$ & $\begin{array}{c}127 \\
(22,23 \%)\end{array}$ & $\begin{array}{c}169 \\
(42,89)\end{array}$ & $\begin{array}{c}57 \\
(14,47)\end{array}$ \\
\hline $\begin{array}{c}\text { Odontología } \\
n=223 \\
(24,13 \%)\end{array}$ & $\begin{array}{c}6 \\
(2,69 \%)\end{array}$ & $\begin{array}{c}15 \\
(6,73 \%)\end{array}$ & $\begin{array}{c}54 \\
(24,22 \%)\end{array}$ & $\begin{array}{c}96 \\
(43,05 \%)\end{array}$ & $\begin{array}{c}52 \\
(23,32)\end{array}$ \\
\hline $\begin{array}{l}\text { Promoción/Prevención de } \\
\text { Enfermedades } \\
n=199 \\
(21,54 \%)\end{array}$ & $\begin{array}{c}5 \\
(2,51 \%)\end{array}$ & $\begin{array}{c}7 \\
(3,52 \%)\end{array}$ & $\begin{array}{c}52 \\
(26,13 \%)\end{array}$ & $\begin{array}{c}102 \\
(51,26 \%)\end{array}$ & $\begin{array}{c}33 \\
(16,58 \%)\end{array}$ \\
\hline $\begin{array}{c}\text { Hospitalización/Cirugía } \\
n=128 \\
(13,85 \%)\end{array}$ & $\begin{array}{c}3 \\
(2,34 \%)\end{array}$ & $\begin{array}{c}13 \\
(10,16 \%)\end{array}$ & $\begin{array}{c}26 \\
(20,31 \%)\end{array}$ & $\begin{array}{c}64 \\
(50 \%)\end{array}$ & $\begin{array}{c}22 \\
(17,19 \%)\end{array}$ \\
\hline $\begin{array}{c}\text { Fisioterapia } \\
\quad n=97 \\
(10,17 \%)\end{array}$ & $\begin{array}{c}1 \\
(1,03 \%)\end{array}$ & $\begin{array}{c}11 \\
(11,34 \%)\end{array}$ & $\begin{array}{c}31 \\
(31,96 \%)\end{array}$ & $\begin{array}{c}30 \\
(30,93 \%)\end{array}$ & $\begin{array}{c}24 \\
(24,74 \%)\end{array}$ \\
\hline
\end{tabular}




\section{DISCUSIÓN}

Los presentes resultados muestran predominio del sexo femenino, similar a lo reportado en Ecuador por Fariño y col (9), Arbeláez y Mendoza (11) y Acosta y col en Argentina (12), pero diferente a los encontrados por Vite y col. en Ecuador donde predominó el sexo masculino (10), las mujeres asisten más veces que los hombres a los centros asistenciales posiblemente por el mayor interés que prestan a su estado de salud, de igual manera se describe que ocupan más las camas hospitalarias, consumen la mayor cantidad de medicamentos prescritos (19) y morbilidad independientemente de las etapas de vida, trabajo y actividades (11).

En cuanto a la edad, en la presente investigación el promedio fue $33 \pm 13,25$ años con un rango entre 20 y 85 años y el mayor grupo estuvo entre $20-39(72,07 \%)$, a diferencia de lo reportado por Acosta y col. (12) que establecieron un rango de edad menor (16-72 años), mientras que González-Quiñones y col, Arbeláez y col., señalan una edad promedio de 37 años y el $88 \%$ menor a 49 años $(11,20)$, mientras que Castelo Rivas establecieron mayor frecuencia de 18 a 24 años (2)

La condición socioeconómica en los resultados actuales más frecuente fue "no pobre", a diferencia de Ortiz-Prado y col. donde las familias con menor ingreso económico tienen un mayor gasto en salud (21) y dificultades para acceder a la atención sanitaria de manera integral y oportuna (22), agravado por la existencia de un presupuesto familiar deficiente destinando, muchas veces, para la adquisición de medicamentos (23), la Organización Panamericana de la Salud establece que el acceso a los servicios de salud difiere según el nivel socioeconómico en pobreza extrema en $10,1 \%$ y $12,3 \%$, con pobreza relativa $14,6 \%$ y 16,8 , y en población no pobre $17,4 \%$ y $21,7 \%(6)$.

En la investigación actual se encontró un predominio de los usuarios que asistían a entidades públicas de salud, como el Ministerio de Salud Pública y el IESS, Institutos de Seguridad de las Fuerzas Armadas y Policía Nacional, entidades privadas como medicina pre-pagada, Atención Privada, resaltando un sistema de salud público y el privado no unificado, donde se atienden a personas pertenecientes a diferentes grupos sociales, pero con barreras de acceso y la coexistencia de diferentes unidades no integradas en la red de salud $(5,6)$.

En cuanto a la presencia de enfermedades crónicas referidas por los usuarios investigados, se evidencia que las más frecuentes son la diabetes mellitus, hipertensión arterial y la obesidad, similar a Cañizares y col., y Villanueva que establecen que la prevalencia estas patologías presentan un rápido aumento en los últimos años, por lo que las instituciones de salud deben adaptarse a los cambios demográficos y epidemiológicos, fomentando la realización de programas educativos para disminuir la morbimortalidad (6,23-26).

Los servicios de atención médica más utilizados por los usuarios estudiados que asistieron a las instituciones de salud en los últimos seis meses, fueron los ejercidos por el médico general, seguido del médico especialista y la realización de estudios en el Laboratorio o radiología, tanto en mujeres como hombres, resultados similares a los referidos por Acosta y col., Pirani y col., Pujol y col. $(12,27,28)$.

En relación al tiempo que debieron esperar los usuarios para ser atendidos por los servicios requeridos, predominó la atención inmediata en medicina general y el tiempo de 1 semana en el servicio de promoción/prevención de enfermedades, estos resultados son diferentes a los descritos por Acosta y col., que el tiempo de espera es aceptable menor a una semana en la mayoría de los casos (12).

La percepción de la calidad de los servicios recibidos en este trabajo la categoría buena predominó en los servicios Programas de Promoción y Prevención, odontología, laboratorio/radiología, similar a Camacho y col., que establecen que a menor tiempo de espera el usuario tendrá una mayor percepción o satisfacción del servicio, incluso, un usuario puede sentirse satisfecho de la atención recibida sin considerar las condiciones de infraestructura de la institución (32).

\section{CONCLUSIONES}

Los presentes resultados muestran que los usuarios investigados en su mayoría son mujeres, predominantemente jóvenes, quienes asisten a los diferentes centros de salud de la Provincia de Manabí-Ecuador preferentemente al MSP y el IESS, estos usuarios presentan enfermedades crónicas que tienen una alta morbi-mortalidad en el país como DM, HTA, la DM, obesidad, infarto al miocardio, artrosis y discapacidad mental principalmente en los adultos de 40-64 años, siendo los servicios más utilizados los proporcionados por el médico general, seguidos del médico especialista, los exámenes realizados en el Laboratorio y los estudios de imágenes en radiología. La percepción que han referido más de la mitad de los usuarios encuestados fue buena, sugiriendo que la atención recibida por parte del personal de salud de los centros de salud de la Provincia de Manabí en Ecuador fue satisfactoria.

FINANCIAMIENTO: no hubo fuentes de financiamiento. CONFLICTOS DE INTERESES: Los autores declaran no tener conflictos de interés.

\section{REFERENCIAS BIBLIOGRÁFICAS}

1. Álvarez F. Gerencia en hospitales e instituciones de salud. Bogotá: Ecoe ediciones; 2013.

2. Castelo Rivas WP, Castelo Rivas AF, Rodríguez Díaz JL. Satisfacción de pacientes con atención médica en emergencias. Rev Cubana Enferm. 2016;32(3):335-342.

3. Chang Campos CJ. Evolución del sistema de salud de Ecuador. Buenas prácticas y desafíos en su construcción en la última década 2005-2014. An Fac med. 2017;78(4):452-60.

4. Organización Panamericana de la Salud/Organización Mundial de la Salud. Informe quinquenal 2013-2017 del Director de la Oficina Sanitaria Panamericana: Capítulo I: Hacia el logro del acceso universal a la salud y la cobertura universal de salud. 2017. (Acceso 20 septiembre 2020). Disponible en: https://www.paho.org/annual-report.2017/Espanol.html\#chapter1. 
5. Bursztyn I, Kushnir R, Giovanella L, Stolkiner A, Sterman-Heimann L, Riveros M, Sollazzo A. Notas para el estudio de la atención primaria en contextos de sistemas de salud segmentados. Rev salud pública. 2010; 12 (1):77-88.

6. Organización Panamericana de la Salud. Perfil de Sistema de Salud: Ecuador, monitoreo y análisis de los procesos de cambios y reforma. $3 .^{\text {a }}$ ed. Washington, DC: OPS; 2008: 44.

7. Constitución de la República de Ecuador. Registro Oficial N449.

8. Lucio R, Villacrés, Henríquez R. Sistema de salud de Ecuador. Salud Públ Méx. 2011; 53: s177-187.

9. Fariño Cortez J, Cercado Mancero A, Vera Lorenti E, Valles Flores J, Ocaña Ocaña A. Satisfacción de los usuarios y calidad de atención que se brinda en las unidades operativas de atención primaria de Salud. Rev Espacios. $2018 ; 39(32): 22$.

10. Vite H, Palomeque I, Romero W. Análisis de calidad del servicio de atención al afiliado en centros de salud del IESS: Caso Santa Rosa y Pasaje. Rev Talentos. 2018;5(2):68-78.

11. Arbeláez G, Mendoza P. Relación entre gestión del director y satisfacción del usuario externo en centros de salud de un distrito del Ecuador. An Fac Med. 2017;78 (2):43-49.

12. Acosta L, Burrone MS, López de Neira MJ, Lucchese M, Cometto $C$, Ciuffolini $B$, et al. Análisis de la satisfacción del usuario en centros de salud del primer nivel de atención en la provincia de Córdoba, Argentina. Enferm Glob. $2011 ; 10$ (1): 21.

13. Borre Y, Vega Y. Calidad percibida de la atención de enfermería por pacientes hospitalizados. Cienc enferm enfermería. 2014; 20(3):81-94.

14. Rey C. La satisfacción del usuario: un concepto en alza. Anales de Documentación. 2000;3:139-53

15. Gutiérrez H. Calidad Total y Productividad. 2da. Ed. Ciudad de México: McGraw Hill; 2005.

16. Instituto Nacional de Estadísticas y Censos (INEC). Coeficientes de GINI por ingresos. Disponible en: https:/www.ecuadorencifras.gob.ec/coeficiente-de-gini-por-ingreos/2.

17. Instituto Nacional de Estadística e Informática. Encuesta Nacional de Satisfacción de Usuarios en Salud 2016. Lima; INEI 2017. (Fecha de Acceso 25 septiembre 2020). Disponible en: https:portal.susalud.gob.pe/wpcontent/uploads/archivo/encuesta-sat-nac/2016/INFORME_FINAL_ENSUSALUD_2016.pdf

18. Ministerio de Salud Pública del Ecuador. Manual del Modelo de Atención Integral del Sistema Nacional de Salud Familiar Comunitario e Intercultural (MAIS.FCI). (Fecha de Acceso 25 septiembre 2020). Disponible en: https://instituciones.msp,gob.ec/somosalud/images/documentos/guia/Manual_MAIS-MSP12. 12.12.pdf

19. Universidad Nacional Autónoma de México. El hombre y la mujer enferman en forma diferente. Boletín de la Academia Nacional de Medicina de México. Rev Fac Med. 2014;57(2):53-56.

20. González-Quiñones JC, Restrepo Chavarriaga G, Hernández-Rojas AD, Ternera-Saavedra D, Galvin-Gómez C; PinzónRamírez J. Satisfacción de pacientes que acudieron al primer nivel de atención en Bogotá. Rev Salud Pública. 2014;16(6):871-884.

21. Ortiz-Prado E, Ponce J, Cornejo-Leon F, Stewartlbarra AM, Trujillo RH, Espín E, et al. Analysis of Health and Drug Access Associated with the Purchasing Power of the Ecuadorian Population. Glob J Health Sci. 2016; 9(1): 201-210

22. Molina Guzmán A. Funcionamiento y gobernanza del Sistema Nacional de Salud del Ecuador. Rev Panam Salud Publica. 2019; 63: 185-205.

23. Cañizares Fuentes R, Mena Ribadeneira G, Barquet Abi-Hanna G. Análisis el sistema de salud de Ecuador. Rev. Med. FCMUCSG. 2015;19(04):193-204.

24. Villanueva M. El proceso administrativo. En Villanueva M. Curso de administración moderna para licenciatura y posgrado. Toluca: Luminanza S.A.; 2011:163-76.

25. Organización Panamericana de la Salud. Metodología de gestión productiva de los servicios de Salud. Manual. Washington D.C.: OPS, 2010.

26. Panamerican Health Organization (PAHO). Health in the Americas 2017. (Fecha de Acceso 12 julio 2020). Disponible en: http://www.paho.org/salud-en-las-americas2017/?post_t_es=ecuador

27. Pirani Gaiosos V, Martins Mishima S. User satisfaction from the perspective off acceptability in the family health scenario. Texto contexto-enferm. 2007; 16(4):617-625.

28. Pujol Rivera E, Gené Badía J, Sans Corrales M, Samprieto-Colom L, Pasarin Rua MI, Igesias-Perez B y col. El producto de la atención primaria definida por profesionales y usuarios. Gac Sanit. 2006; 20(3):209-219.

29. De los Ríos Castillo JL, Ávila Rojas TL. Algunas consideraciones en el análisis del concepto: satisfacción del paciente. Invest educ enferm. 2004; XXII (2):128-137.

30. Prado Kantorski L, Jardim VR, Wetzel C, Olschowsky A, Schneider JF, Heck RM y col. Satisfação dos usuários dos centros de atenção psicossocial da região Sul do Brasil. Rev Saúde Pública. 2009; 43: s29-35

31. Jaegger RC, Guitton AP, Lyrio JM, Santos MM, Freitas RC, Gonçalves SR et al. A experiência de morar fora: avaliação da satisfação de usuários em um serviço de saúde mental. Cad saúde Colet. 2004; 12(1):27-39.

32. Camacho F, Anderso R, Safrit A, Jones AS, HoffmannP. The relationship between patient's perceived waiting time and officebased practice satisfaction. N Med J. 2006;67(6):409-13 